\title{
Klinische Funktionsanalyse Manuelle Strukturanalyse • Interdisziplinäre Diagnostik
}
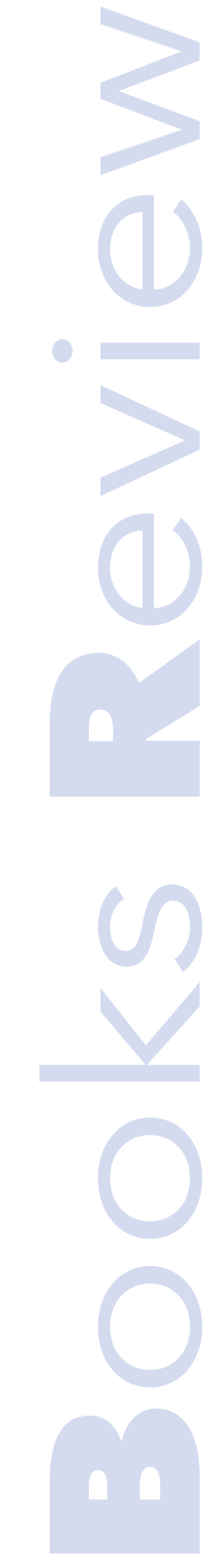

Marian-Vladimir Constantinescu $\mathrm{DDS}, \mathrm{PhD}$ Department of Prosthetic Dentistry "Carol Davila" University of Medicine and Pharmacy Bucharest, Romania Email:

drvladimir.constantinescu@gmail.com
M. Oliver Ahlers, Holger A. Jakstat

Publisher: dentaConcept

Language: German

ISSN: 978-3-933465-03-0

Edition: 4/e

Publish Year: 2011

Pages: 681

Price: $168,00 €$

The book "Clinical Functional Analysis Manual Structural Analysis - Interdisciplinary Diagnostics" by M. Oliver Ahlers and Holger A. Jakstat presents a conceptual idea of an efficient diagnosis scheme for a contemporary dental practice of the Craniomandibular Dysfunction (CMD). It is a practical workbook which explains the Craniomandibular Disorders (CMD) and their diagnosis under an interdisciplinary approach.

The currentedition, the 4th, of this bookis divided into six chapters: Clinical Functional Analysis, Dysfunction of the Masticatory System, Dental Clinical Functional Analysis, Interdisciplinary Cooperation, Instrumental Function Diagnostics and Therapy, and Management in Treatment of Functional Diagnostics. The first chapter shows the importance of functional diagnostics and updated changes in diagnosis and treatment in Craniomandibular Disorder (CMD).

Chapter two enlarges upon the epidemiology, classification and terminology, functional anatomy, etiology and pathology, pain syndrome and therapy orientation in dysfunctions of the masticatory system.

The need for functional dental clinical analysis (CMD-Screening), medical history, stress analysis, pain analysis and observation of disease progression are presented in chapter three. With respect to the clinical functional examination, the authors point out the role of Manual Structural Analysis (vertical/horizontal isometry, passive/dynamic compression, translation and traction) in the Craniomandibular Disorder (CMD) diagnosis. Functional computer analysis includes (CMDfact) modules for manual structural analysis ("CMDmanu") and for the evaluation and measurement of TMJ MRIs ("CMDtomo") as well as instrumental functional analysis CMD 3D.

Chapter 4 speaks about the psychological factors and orthopedic influences that contribute to CMD occurrence in an interdisciplinary approach of the examination and treatment of this disease. Tinnitus and otalgia are presented, as well as the imaging investigation procedure, physiotherapy, treatment options of acute and chronic pain, and conversation in CMD patients.

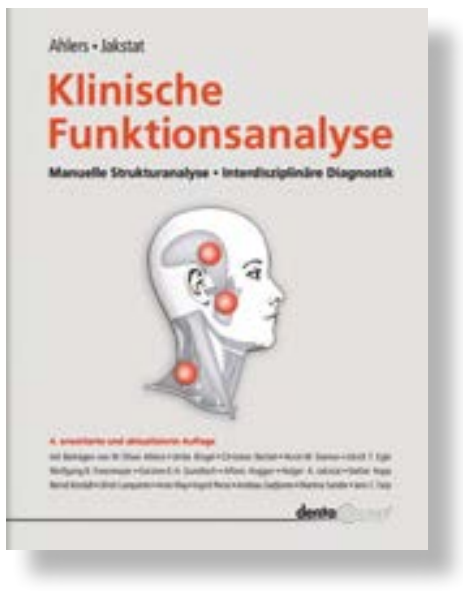

Chapter five describes the instrumental function diagnostics and occlusal therapy beginning with the clinical examination of the upper and lower dental arches. After an alginate precision impression is achieved a class 4 gypsum study model is cast. Then register cranial landmarks are gathered to position the upper model. Then there follows the transfer of the cranial landmarks on the articulator and installation of the lower model. The static bite registration fits the checking of the models in the articulator.

Then the kinematic registration of the eccentric positions is considered. The clinical data recorded explains how to adjust the articulator and analyze the condylar position with electronic measuring devices.

Further data present the splint therapy, different types of splints, the difference between them and the use of the rails in the craniomandibular disorders.

In chapter six the management of functional diagnostics is illustrated insisting on the functional diagnosis in daily practice, the systematic documentation of quality control and the importance of informing patients about the role of the functional analysis. The contribution of the functional analysis in the dental diagnosis is emphasized as well as the digital data transfer in the clinic.

The book "Clinical Functional Analysis Manual Structural Analysis • Interdisciplinary Diagnostics" by M. Oliver Ahlers and Holger A. Jakstat of 681 pages is richly illustrated to interact with the readers.

Reviewing this book we have the opportunity to inform you about the latest news in diagnosis and treatment of craniomandibular functional disorders for the contemporary dental practice under an interdisciplinary approach. 